Case report

\title{
Renal cell carcinoma with bilateral synchronous adrenal gland metastases: a case report Georgios E Koutalellis ${ }^{1 *}$, Evangelos Felekouras ${ }^{2}$, Constantinos Evangelou ${ }^{3}$, Georgios Koritsiadis ${ }^{1}$, Dimitrios Chasiotis ${ }^{2}$ and Ioannis Anastasiou ${ }^{1}$
}

\author{
Addresses: ${ }^{1}$ Department of Urology, "Laiko" General Hospital, Athens Medical School, 17 Agiou Thoma str, 11527, Goudi, Athens, Greece \\ ${ }^{2}$ Department of Surgery, "Laiko" General Hospital, Athens Medical School, 17 Agiou Thoma str, 11527, Goudi, Athens, Greece \\ ${ }^{3}$ Department of Pathology, "Laiko" General Hospital, Athens Medical School, 17 Agiou Thoma str, 11527, Goudi, Athens, Greece \\ Email: GEK* - gkoutalellis@yahoo.com; EF - felek@med.uoa.gr; CE - costasevagelou@hotmail.com; GK - koritsiadisdc@yahoo.gr; \\ DC - Dimitrischasiotis@yahoo.gr; IA - ekati2@otenet.gr \\ * Corresponding author \\ Received: 16 April 2009 Accepted: 18 August 2009 Published: 9 September 2009 \\ Cases Journal 2009, 2:7298 doi: 10.4076/1757-1626-2-7298 \\ This article is available from: http://casesjournal.com/casesjournal/article/view/7298 \\ (C) 2009 Koutalellis et al.; licensee Cases Network Ltd. \\ This is an Open Access article distributed under the terms of the Creative Commons Attribution License (http://creativecommons.org/licenses/by/3.0), \\ which permits unrestricted use, distribution, and reproduction in any medium, provided the original work is properly cited.
}

\begin{abstract}
Introduction: Renal cell carcinoma is characterized by its potential of metastasizing widely and to unusual sites, with the metastases occasionally preceding clinical recognition of the primary tumor. Synchronous bilateral adrenal metastases from renal cell carcinoma, without other metastases, are rare and, to our knowledge, only 17 cases have been published in the literature to date. In general, patients with synchronous bilateral adrenal metastases from renal cell carcinoma have a poor prognosis.

Case presentation: We report a case of right-sided renal cell carcinoma with simultaneous bilateral adrenal metastases in a 58-year-old woman. The primary tumor was localized in the upper and mid pole of the kidney. The diagnosis was established preoperatively by abdominal ultrasound and computed tomography. Surgical treatment consisted of a right radical nephrectomy and bilateral adrenalectomy. Postoperative cortisone acetate replacement was instituted. The pathological findings of the right renal tumor showed clear cell carcinoma and both adrenal tumors showed the same pathology as the right renal tumor. There was no evidence of recurrence after 6 months of follow-up.

Conclusion: Patients with bilateral synchronous adrenal metastases should be considered to have disseminated metastatic disease. However, good performance status, the presence of paraneoplastic syndrome and the alleviation of refractory pain are important reasons make an urologist to consider radical nephrectomy in renal cell carcinoma patient with metastases.
\end{abstract}

\section{Introduction}

It is well established that the incidence of adrenal metastases in certain types of malignancy, such as carcinomas of the breast, lung and melanoma is high [1]. Renal cell carcinoma (RCC) can metastasize extensively and to unusual sites. Due to the insidious clinical course of many primary RCCs, approximately a third of all patients have metastases at the initial diagnosis. The most common metastatic sites from RCC are the lung, lymph nodes, liver, bone, contralateral kidney and 
ipsilateral adrenal gland [2]. The incidence of adrenal metastases from RCC is 6-29\% in autopsy series [3-5], and one third of this incidence (2-10\%) from clinical diagnosis [6-9]. Synchronous bilateral involvement was found to be present at about one fifth of all adrenal metastases $[1,10]$. Adrenal metastases from RCC are either synchronous or metachronous. In this article, we report a case of rightsided RCC with simultaneous bilateral adrenal metastases, the treatment and prognosis are also discussed.

\section{Case presentation}

A 58-year-old Caucasian-Greek woman presented with a dull pain located in the right flank for 4 years. Other symptoms, including hematuria, were not present. The patient had a medical history of spondylolisthesis, osteoporosis, hypertension and hyperlipidemia. The gradual deterioration of pain in the last month before her admission caused her to seek medical attention. The pain initially was attributed to spondylolisthesis and she was prescribed a relative medication. Due to the persistence of pain despite the administration of the specific medication she was submitted to sonography. The ultrasonography revealed a right upper pole renal mass and bilateral adrenal masses. Because of these findings she was admitted for further evaluation. A physical examination revealed a mild tenderness in her right flank. No lymphadenopathy was found. The patient's blood pressure and the pulse rate were normal. A contrast-enhanced abdominal computed tomography (CT) confirmed the presence of a $6 \mathrm{~cm} \times 5 \mathrm{~cm} \times 4 \mathrm{~cm}$ solid mass at the upper and mid pole of the right kidney with heterogeneous and enhanced areas and bilateral involvement of the adrenal glands (Figure 1). A chest $\mathrm{x}$-ray, chest CT scan, and bone scan were all negative for metastases. Laboratory tests revealed no abnormalities. Endocrinological evaluation demonstrated normal serum and urinary levels of aldosterone, cortisol and 17-hydroxysteroids. The diagnosis of right renal cell carcinoma with bilateral adrenal metastases was established.

Right radical nephrectomy was performed through a bilateral subcostal incision (Chevron). The contents of Gerota's fascia were removed en bloc, followed by a total left adrenalectomy. Thorough exploration of the abdominal cavity did not disclose any other metastatic focus.

Histological examination of the resected specimens revealed a solid tumor with golden-yellow appearance, relatively well circumscribed and measuring $5.6 \times 4.8 \times$ $3.5 \mathrm{~cm}$ in the upper half of the right kidney. Almost the entire left adrenal gland, measuring $6.2 \times 3.1 \times 2.7 \mathrm{~cm}$, was occupied by a brownish compact tumor while only a thin rim of adrenal tissue was preserved. A same mass, with a maximum diameter of $1.9 \mathrm{~cm}$, was also observed in the right adrenal gland. Microscopic examination of the renal and both adrenal gland tumors revealed the diagnosis of

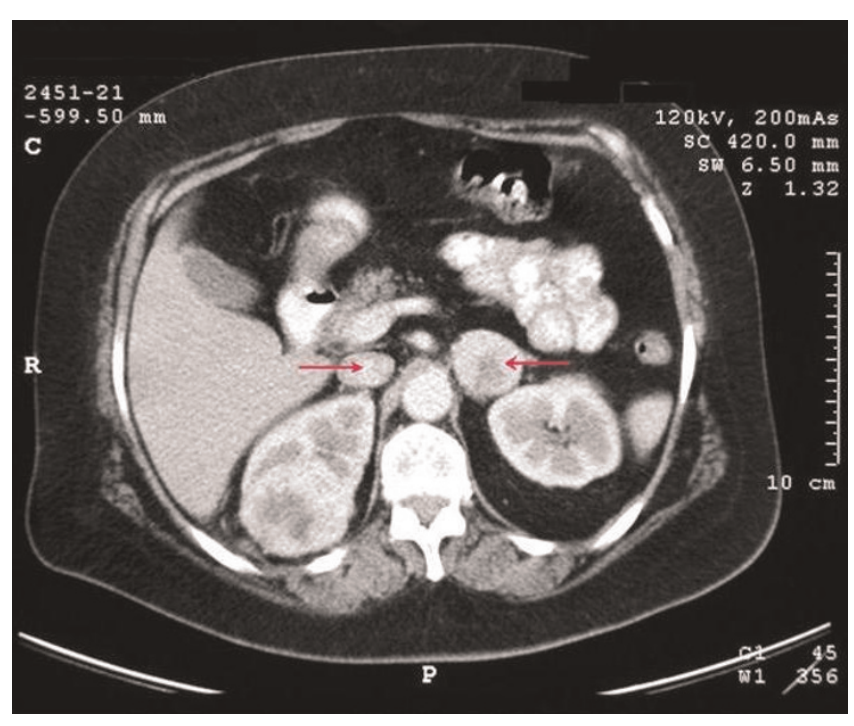

Figure I. Contrast-enhanced abdominal computed tomography (CT) revealed a $6 \mathrm{~cm} \times 5 \mathrm{~cm} \times 4 \mathrm{~cm}$ solid mass at the upper and mid pole of the right kidney that consisted of heterogeneous and enhanced areas and also bilateral involvement of the adrenal glands (arrows).

clear cell renal carcinoma, Grade3 and pT3 according to the TNM classification system (Figure 2). The carcinoma invaded and focally disrupted the renal pelvis. Invasion of the perinephric fat to a limit extend as well as of various vessels by neoplastic cells was also observed.

Convalescence was uneventful. The dosage of cortisone was gradually tapered to $12.5 \mathrm{mg}$ per day orally. Serum

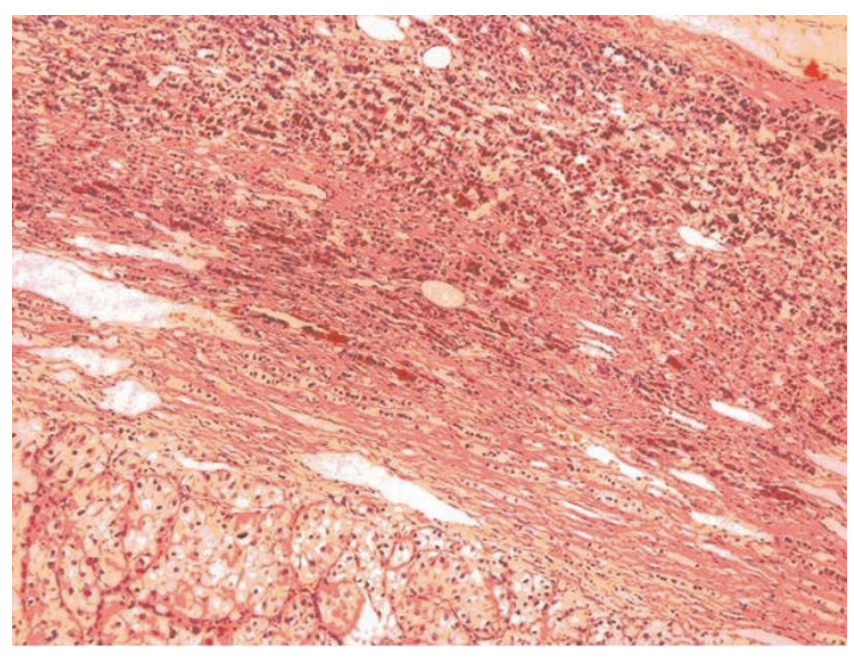

Figure 2. Histological section of clear RCC metastasis in the left adrenal gland. Only a thin rim of adrenal tissue is preserved (H \& E stain, 200X). 
cortisol and electrolytes were normal during the follow-up period. She was discharged 10 days after the operation.

The patient is alive with no evidence of disease recurrence or metastatic disease after six months postoperatively treated with adjuvant chemotherapy.

\section{Discussion}

Adrenals involvements are usually silent because metastatic lesions fail to destroy enough tissue to produce clinical signs or symptoms of insufficiency. However, adrenal function is usually maintained even though bilateral adrenal glands are affected by malignancy. There is a higher incidence of upper pole primary renal tumors than either mid or lower pole renal tumors in patients with adrenal metastases [11]. Patients with adrenal metastases tend to have large primary renal tumors that nearly encompass the entire kidney [11]. Adrenal metastases result from direct extension of the renal tumor, lymphatic spread from involved retroperitoneal nodes, retrograde venous embolization and local vascular spread through vessels within Gerota's fascia [12].

The presence of ipsilateral adrenal involvement appears to portend a poor prognosis. These patients have high-stage primary tumors (T3 or greater). Antonelli et al. [10] concluded that ipsilateral adrenal sparing procedure is only safe during surgery for organ-confined RCC of $\leq 4 \mathrm{~cm}$, while the location of cancer according to preoperative CT findings cannot fully predict the risk of ipsilateral metastases. Moreover, any suspicious contralateral adrenal mass should be explored because there is a high probability of removing a metastasis. In an another study, authors recommended ipsilateral adrenalectomy in conjunction with radical nephrectomy for RCC being indicated in case of large, upper pole extracapsular lesions; even then, it does not affect prognosis [13].

Patients with RCC and a single contralateral adrenal metastasis should be considered as having a solitary metastasis. They should undergo radical nephrectomy and contralateral adrenalectomy. Ipsilateral adrenalectomy should be performed if there is suspicion of metastasis [14]. These patients appear to have better survival than those with bilateral adrenal metastases.

Patients with bilateral synchronous adrenal metastases from RCC should be considered to have disseminated metastatic disease. There are only 18 cases of bilateral adrenal metastases from RCC, without other metastases, that have been reported in the literature, including our case. The follow-up duration is less than 4 years. Despite prophylactic treatment after the operation with chemotherapy and immunotherapy the prognosis remains poor.
An alternative treatment option in cases of RCC with bilateral adrenal metastases is partial adrenalectomy, sparing the normal adrenal tissue [15]. This procedure results in the preservation of the adrenal function while decreasing the tumor burden. In such a manner, steroid replacement and its side effects such as gastritis, hypertension, hypokalemia and Addisional crises during stressful periods can be avoided.

However, the goal of any surgical or medical procedure should be to improve the quality of life and duration of survival. Radical nephrectomy in RCC patients with metastases may be considered in the presence of paraneoplastic syndrome provided the patient is of good performance status. Surgical intervention may also be indicated for palliative reasons. In our case, the patient hadn't paraneoplastic syndrome, was in good performance status, and the surgical intervention performed for the alleviation of severe, refractory pain.

\section{Conclusions}

In our opinion, some patients with bilateral adrenal metastases from RCC and without metastases in other site may be benefited by radical operation, as regards their overall survival, because the reduction of the tumor burden may enhance response rates to postoperative adjuvant therapy.

\section{Abbreviations}

CT, computed tomography; RCC, renal cell carcinoma.

\section{Consent}

Written informed consent was obtained from the patient for publication of this case report and any accompanying images. A copy of the written consent is available for review by the Editor-in-Chief of this journal.

\section{Competing interests}

The authors declare that they have no competing interests.

\section{Authors' contributions}

GEK collected the data and literature review, and wrote the manuscript. EF and IA were the attending doctors, carried out the surgical procedure and literature review. CE performed the pathological examination and assisted in writing the report. DC and GK revised and provided comments on the manuscript.

\section{Acknowledgements}

The authors state that there was no extra institutional funding.

\section{References}

I. Zornoza J, Bernardino ME: Bilateral adrenal metastases. 'Head light' sign. Urology 1980, I 5:91-92. 
2. Duggan MA, Forestell CF, Hanley DA: Adrenal metastases of renal-cell carcinoma 19 years after nephrectomy. Fine needle aspiration cytology of a case. Acta Cytol 1987, 31:5 I2-5I6.

3. Saitoh H, Nakayama M, Nakamura K, Satoh T: Distant metastasis of renal adenocarcinoma in nephrectomized cases. J Urol 1982, 127:1092-1095.

4. Hellsten S, Berge T, Linell F: Clinically unrecognised renal carcinoma: aspects of tumour morphology, lymphatic and haematogenous metastatic spread. $\mathrm{Br}$ J Urol 1983, 55:166-170.

5. Hajdu SI, Thomas AG: Renal cell carcinoma at autopsy. J Urol 1967, 97:978-982.

6. Siemer S, Lehmann J, Kamradt J, Loch T, Remberger K, Humke U, Ziegler M, Stöckle M: Adrenal matastasis in 1635 patients with renal cell carcinoma: outcome and indication for adrenalectomy. J Urol 2004, I 7 I (6 Pt I):2155-2I59.

7. Angervall L, Wahlqvist L: Follow-up and prognosis of renal carcinoma in a series operated by perifascial nephrectomy combined with adrenalectomy and retroperitoneal linfoadenectomy. Eur Urol 1978, 4:13-17.

8. Robey EL, Schellhammer PF: The adrenal gland and renal cell carcinoma: is ipsilateral adrenalectomy a necessary component of radical nephrectomy? J Urol 1986, I35:453-455.

9. Paul R, Mordhorst J, Leyh H, Hartung R: Incidence and outcome of patients with adrenal metastases of renal cell cancer. Urology 200I, 57:878-882.

10. Antonelli A, Cozzoli A, Simeone C: Surgical treatment of adrenal metastasis from renal cell carcinoma: a single-centre experience of 45 patients. BJU Int 2006, 97:505-508.

II. Sagalowsky A, Kadesky K, Ewalt D, Kennedy T]: Factors influencing adrenal metastasis in renal cell carcinoma. J Urol 1994, I5 I: I I8I-II 84.

12. Kozlowski J: Management of distant solitary recurrence in the patient with renal cancer. Contralateral kidney and other sites. Urol Clin North Am 1994, 2 I:60I-624.

13. Sandock DS, Seftel AD, Resnick MI: Adrenal metastases from renal cell carcinoma: role of ipsilateral adrenalectomy and definition of stage. Urology 1997, 49:28-31.

14. Previte S, Willscher M, Burke C: Renal cell carcinoma with solitary contralateral metastasis: experience with 2 cases. J Urol 1982, I 28:132-134.

15. Schomer NS, Mohler JL: Partial adrenalectomy for renal cell carcinoma with bilateral adrenal metastases. J Urol 1995, I53:1196-1198.

\section{Do you have a case to share?}

Submit your case report today

- Rapid peer review

- Fast publication

- PubMed indexing

- Inclusion in Cases Database

Any patient, any case, can teach us something

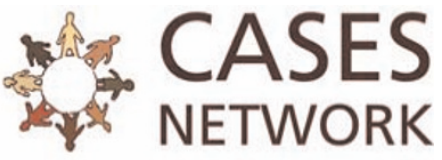

www.casesnetwork.com 